\title{
A Review on Design and Development of Performance Evaluation Model for Bio- Informatics Data Using Hadoop
}

\author{
Ravi Kumar A ${ }^{\text {a }}$, Dr. Harsh Pratap Singh ${ }^{\text {b }}$ and Dr. G.Anil Kumar ${ }^{\mathrm{c}}$ \\ ${ }^{a}$ Research Scholar, Dept. of Computer Science \& Engineering, \\ Sri Satya Sai University of Technology \& Medical Sciences, Sehore, Bhopal Indore Road, Madhya Pradesh, India \\ ${ }^{\mathbf{b}}$ Research Guide, Dept. of Computer Science \& Engineering, \\ Sri Satya Sai University of Technology \& Medical Sciences, Sehore, Bhopal Indore Road, Madhya Pradesh, India \\ ${ }^{c}$ Research Co-Guide, Dept. of Computer Science \& Engineering,Science Institute of Technology,Ibrahim Patnam, Hyderabad
}

Article History: Received: 11 January 2021; Accepted: 27 February 2021; Published online: 5 April 2021

\begin{abstract}
The paper reviews the usage of the platform Hadoop in applications for systemic bioinformatics. Hadoop offers another system for Structural Bioinformatics to break down broad fractions of the Protein Data Bank that is crucial to highthroughput investigations of (for example) protein-ligand docking, protein-ligand complex clustering, and structural alignment. In specific, we review different applications of high-throughput analyses and their scalability in the literature using Hadoop. In comparison to revising the algorithms, we find that these organisations typically use a realized executable called MapReduce. Scalability demonstrates variable behavior in correlation with other batch schedulers, particularly as immediate examinations are usually not accessible on a similar platform. Direct Hadoop examinations with batch schedulers are missing in the literature, but we note that there is some evidence that the scale of MPI executions is better than Hadoop. The dilemma of the interface and structure of an asset to use Hadoop is a significant obstacle to the utilization of the Hadoop biological framework. This will enhance additional time as Hadoop interfaces, such as enhancing Flash, increasing the use of cloud platforms, and normalized approaches, for example, are taken up by Workflow Languages.
\end{abstract}

KEYWORDS: Structural Bioinformatics, Hadoop, Cloud Computing, MapReduce, Spark, Big data

\section{Introduction}

Late advancements in molecular biology and genomics have contributed to significant innovations in digital biological knowledge. This broad measure of data is typically dissected through rehashed use of relatively parallel algorithms: model settings include sequence matching, consistency articulation analysis, and QTL (Quantitative Feature Locus) inquiry and haplotype reconstruction. As of now, we are developing a suite of bioinformatics applications, Bio Hadoop, which illustrates how general-purpose parallelization innovation can be easily and effectively tailored to solve this class of high-performance problems and, more specifically, how this innovation can be the premise of a distributed computing framework for a few computational biology issues. MapReduce is a simple-to-use, general-purpose parallel programming model custom-made for massive dataset scanning on a bunch of item equipment. Engineers only need to write two capacities: a map that blends an info key/esteem pair to a bunch of intermediate keys/esteems, and a Reduces map that consolidates all the intermediate qualities correlated with a specified intermediate key. The structure naturally manages all low-level subtilities such as data partitioning, routing, load balancing, system malfunction handling, and inter-machine contact.

The Apache Hadoop project is a software environment, e.g. an array of interrelated, collaborative projects that shape a standard creative framework to break down vast data sets. Hadoop provides three planned focus points for the investigation of broad collections of biological data. In the primary case, it is intended for the analysis of broad semi-organized data sets; furthermore, it is intended to be a shortcoming of open mindedness (basically by guaranteeing an extremely large measure of general repetition) which turns out to be literally inevitable for a sufficiently large number of processors; finally, MapReduce formalism for the representation of data sets considers $t$ Considering the relevance of computationally analysing protein structures in fields such as membrane proteins, protein-protein interactions, and their effect in system biology and protein architecture of a platform for the successful investigation of semi-organized data sets. For eg, those contained in the Protein Data Bank on a broad scale will be a big advance forward. Then again, Hadoop further outlines the limitations of its selection within the Bioinformatics network and the analysis of structural results. In the key case, Hadoop is a progression of Java libraries, and henceforth there is an assumption that knowledge can be consumed by every bioinformatician or systemic biologist who has not used Java before, however we notice that later increases to the Hadoop ecosystem, e.g., Spark, provide a broader variety of languages. Correspondingly, not at all like dataparallel languages, for example, High-Performance Fortran, Hadoop cannot be readily retrofitted to a steady code base regardless of if the first code is written in Java, although it is feasible to use Hadoop to express occurrences of an executable identical to those of the batch schedulers. Last but not least, the implementation of Hadoop (and along these lines of Spark) in a community is not inconsequential and needs a crucial degree of competence from an essential framework supervisor. As we remember, this last challenge is being dealt with on cloud systems, for example, Azure and AWS. 


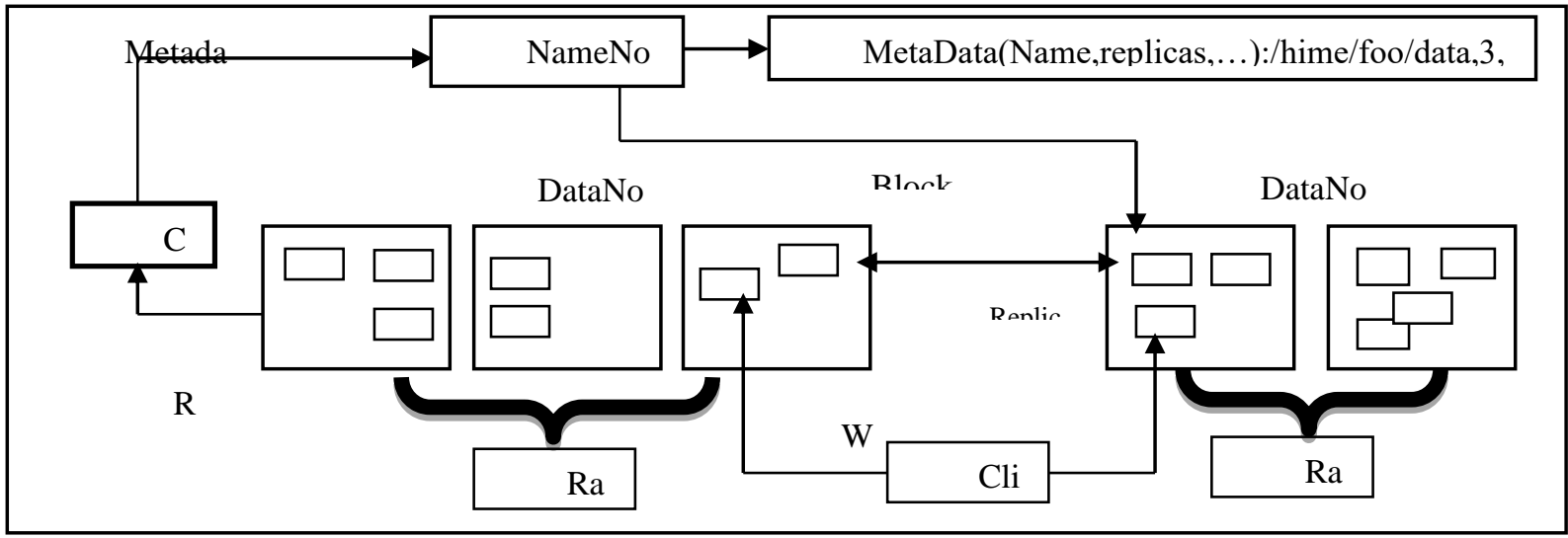

Figure 1 Hadoop Distributed File System

This paper discusses the diversity of work done mainly in Bioinformatics and Systematic Bioinformatics on Hadoop. In particular, relative to other methodologies, such as batch schedulers and MPIs, this paper would evaluate how stable these platforms are. The bulk of this essay is structured in the following manner. A brief review of the Hadoop and Spark structures and a portrait of the batch schedulers and the MPI in the main event. At that point, it depicts Hadoop formalism. A concise survey of the usage of Hadoop in Bioinformatics is accompanied by an internal and external examination of the use of Hadoop in Systemic Bioinformatics. In specific, we discuss the variety of contexts in which Hadoop has been used, the scaling up of those structures in the analysis of various platforms, where it is available and its dependency on the internal structure of the type of Hadoop used. This paper would eventually make a few inferences regarding the scalability of Hadoop and its use in structural bioinformatics.

\section{HADOOP'S USES IN BIOINFORMATICS}

Specialists in various bioinformatics regions have not underestimated the emergence of platforms utilizing the Hadoop infrastructure that we researched, in particular Hadoop and Spark. Several organizations within the Apache Hadoop ecosystem have discovered valuable bioinformatics technologies. This includes the Hive datawarehousing framework, which has the query language of the SQL style, the high-level data-stream language Pig, which gathers material via the execution venture sequences of MapReduce Hadoop, the Mahout machinelearning and clustering offices, and the distributed, scalable database HBase. These companies use the Hadoop Bulk Infrastructure and the Distributed Paper Network, and then take advantage of their plan's scalability and fault-tolerance, as mentioned above.

\section{MapReduce}

MapReduce is a Java-dependent planning technique and programme model for distributed computing. The estimation of MapReduce comprises of two major undertakings, in particular Map and Reduce. The chart takes a bunch of data and transforms it to a different data collection, where the singular components are divided into tuples (key/steem sets). In addition, minimise the job, which takes the output from the map as a details and joins the data tuples in a more modest tuple structure. As the MapReduce name series infers, the reduced activity is continuously done after map work. The important desired role of MapReduce is that it is anything but challenging to scale data preparation across multiple computing hubs. Under the MapReduce scheme, native data handling is referred to as mappers and reducers.

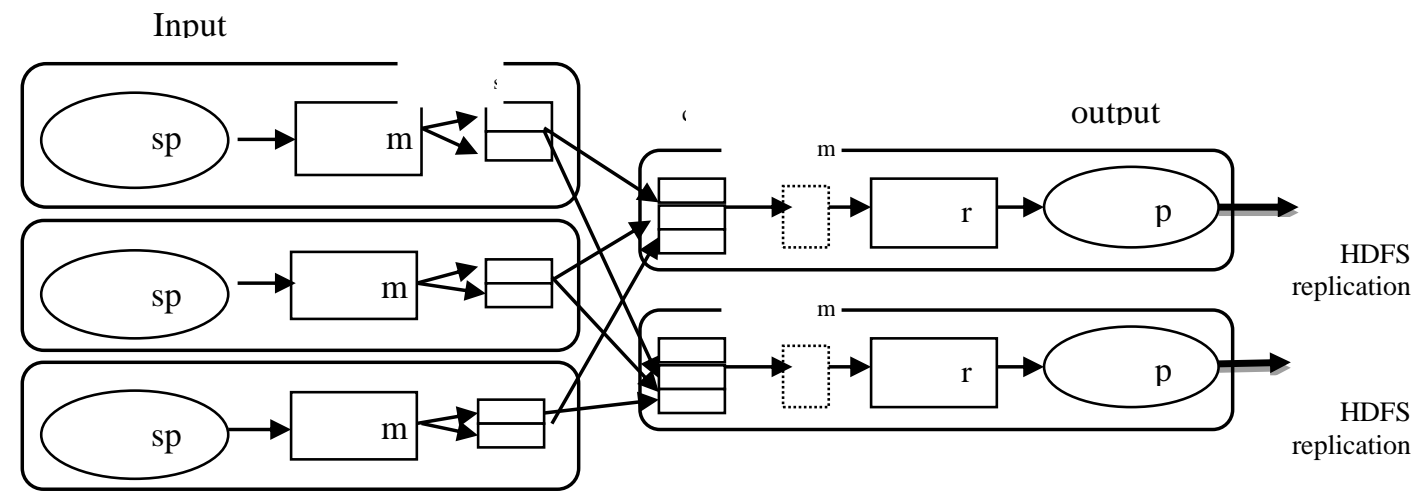

Figure 2 Hadoop MapReduce 
Breaking down the data handling programme into mappers and reducers is often non-trivial. However, when we write a programme in the MapReduce structure, scaling the application to operate more than a hundred, thousands, or even a massive amount of machines in a bunch is just a configuration adjustment. This simple scalability is what has motivated a huge number of software engineers to use the MapReduce model. As far as software programmes are concerned, MapReduce has been used for a number of problems in managing biological and sequencing datasets. Some of the extraordinary ventures in the sequence alignment area are Cloudburst and Cloud Aligner, all of which rely on the RMAP alignment measurement, and Cloud Blast, which depends on the BLAST calculation. It is crucial that MapReduce can be especially suitable for, for example, the creation of the de Bruijn table, so that the genome can be reunited all over again. For eg, Contrail will compile contiguousness records for all kmers in the genomic sequence of peruses and then use dispersed conglomeration capacities, e.g. lower to pack simple chains of length $\mathrm{N}$ in $\mathrm{O}(\log (\mathrm{N}))$ adjusts using randomised simultaneous overview positioning measurements. Devices are often performed in MapReduce for the analysis of amassed sequencing results, e.g. Crossbow is intended for the detection of GSP (Single Nucleotide Polymorphism). It makes use of the Bowtie and the SPS guest SOAPsnp. Differential articulation (using RNASeq) may be calculated using the Myrna software pipeline-the pipelines are data streams comprising successive phases through which the bioinformatics software is applied to the data. In addition, the Java-based Genome Analysis Toolkit (GATK) libraries generated by the Wide Institute and the Hadoop-BAM, much like the SparkSeq Scalabased library, have appeared in numerous programming libraries that facilitate the management and preparation of sequencing data document configurations, such as the SAM Sequence Alignment Map and the BAM (Binary Alignment Map) (talked about beneath). GATK provides the data capabilities of the board as data access designs, is separated from higher-level capabilities in specific, low-level use, and also gives the ability of the board to examine figures. In addition, the Workflow Definition Language (WDL) was created by the Large Institute for use in data investigation pipelines (examined in the following segment). It is a language of a high level which is supposed to be understandable and scriptable. It allows researchers to portray survey undertakings, daisy-chain transfers into workflows, and to use specialized highlights, such as parallelization. As a consequence of the need for standardisation across the various pipeline arrangements, WDL was developed, giving a widespread standard. The execution engine is critical in order to conduct test pipelines written in WDL. Cromwell is such an engine that WDL engineers are also planning to operate on a variety of platforms (local, HPC or Google-maintaining multiple platforms, such as Microsoft Azure and AWS) and can respond to workflow needs flexibly. In addition, other striking workflow languages, such as CWL (Common Workflow Language) and Next Flow, are pushing forward and are beginning to pick up footholds that are built to promote reproducible workflows. CWL is sponsored by Cromwell, Galaxy and Tavern, targeting Bioinformatics, Clinical Imaging, Chemistry, Physics and Astronomy. The representation of the basic platform through the excellence of the execution engine that can operate and parallel workflow occupations on platforms such as LSF, SLURM and AWS is taken into account by Next flow, which relies on the Groovy vocabulary. In addition, the agreement for the pipeline upgrade specifically for the Hadoop framework is usable. For example, SparkSeq is a library for the creation of pipelines for genomic research using Apache Spark's Scala. While Scala is supported by the Spark framework, it does not have a comparable bioinformatics client base, as it values data research and deep learning networks. The aim of these devices is to abuse the scalability provided by the Hadoop and Spark platforms in view of the massive data sequence measures being constructed, and this balances any difficulties in designing or re-composing those applications. For eg, though, the development in general standards may be that WDL provides scientists with a more easy-to-understand way to use methods designed for those platforms.

\section{Spark}

Apache Spark is an incredibly fast community computing invention built for simple computation. Depends on Hadoop MapReduce and expands the MapReduce paradigm to allow efficient use of it for additional forms of calculations, including dynamic queries and stream management. Spark's key highlight is its in-memory community computing, which speeds up the programme. Spark is developed to cover a broad variety of workloads, such as batch processes, iterative algorithms, collaborative requests, and streaming. In addition to supporting each of these workloads in a single setting, the administration weight of having the resources separated is minimised. For the Apache Spark system directly, multiple implementations have been improved. A broad audit of the bioinformatics applications that use Spark has been carried out by Guo et al. An HDFS model genomics pipeline in Spark has been developed by Nothaft et al. Compared with the equal pipeline operating on existing instruments, they have seen performance gains on the platform running on Amazon EC2. Their pipeline includes synchronisation of peruses, pre-handling of QC peruses, calling for variants, and filtration of optimistic variance calls. ADAM pipeline phases were measured against other important instruments used for these purposes, such as GATK, SAM and Sambamba. They gained super linear speedup when expanding from $8 \mathrm{x}$ to $16 x$ hubs, and shut down straight speedup while scaling from $32 x$ to $128 x$ hubs. This led to a $63 \%$ reduction in execution costs on the Amazon EC2. Variation Spark is a machine learning analysis application for modified genomic data using Apache Spark. It functions on broad part vectors and a large range of genetic 
characterization measures and can conduct continuous examinations. Right now, Variant Spark uses k-implies clustering, but the subsequent rendering will change an unusual backwood calculation). The Genome Analysis Toolkit (GATK), commonly used in Variant Disclosure, offers a portion of its devices modified in Spark-the past adaptations of the Java libraries to Hadoop. Although Spark's GATK devices may be a sudden spike in demand for a solitary Spark hub, efficiency improvements are produced because the Spark network is a bunch of process hubs that are scaled up on a level plane. Although there are a large number of applications that use Spark in the field of genomics, there are actually few applications in the field of structural bioinformatics. MTTF is another minimized parallel machine design for PDB data and Hadoop and Spark are eligible for analysis with a single Hadoop data selection sequence record.

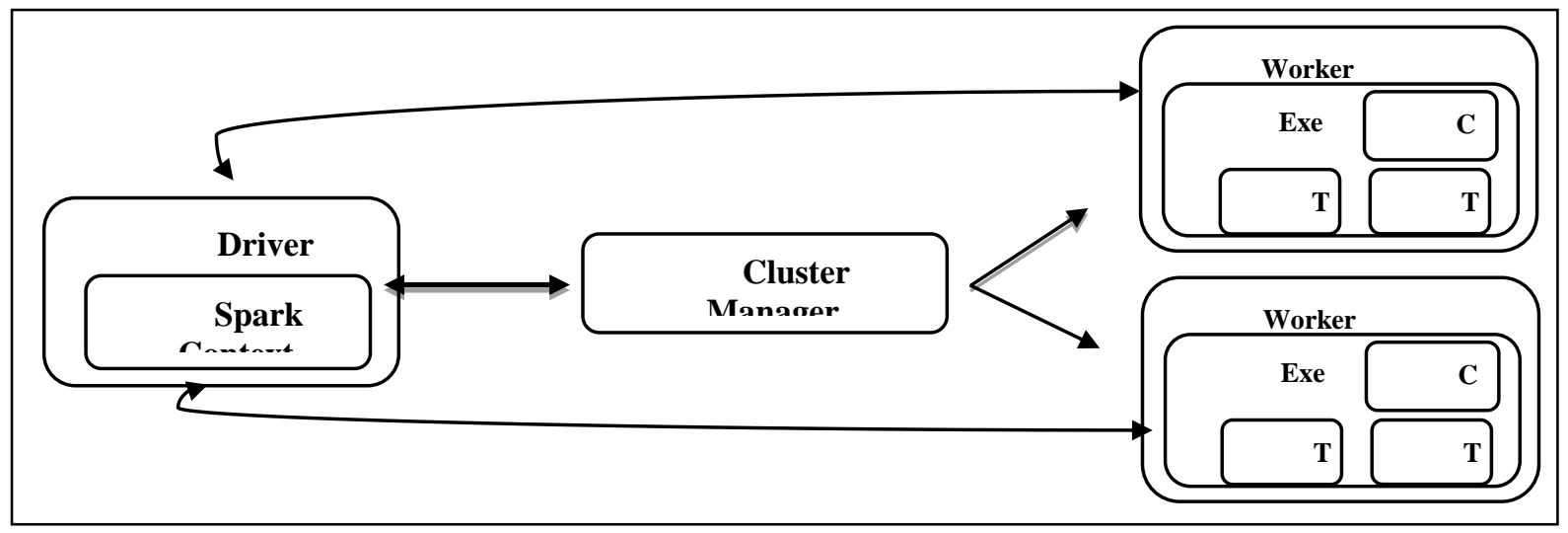

Different reports, for example, illustrate preliminary arrangements to modify Spark, but as of now there are no different instances of Spark being introduced to systemic bioinformatics.

\section{LITERATURE REVIEW}

In biomedical and health sciences, large-scale data technologies are rapidly being utilized. Large biological and clinical data measurements have been developed and collected at exceptional speed and scale. The new age of sequencing advancements, for instance, requires billions of DNA sequence data to be analyzed on a daily basis, and the utilization of electronic health records (EHRs) reports large patient data measurements. With the support of revolutionary redesigns such as the rise of advanced sequencing systems, the creation of new parallel processing equipment and applications and the large growth of EHRs, the cost of obtaining and dissecting biomedical data is expected to decrease significantly. Wide networks in technologies provide innovative ways of finding new knowledge and developing revolutionary ways to transform the essence of health care. A constantly changing field is the use of large data in health care, with many recent disclosures and programs scattered across the past five years. The usage of large data in four major biomedical sub-disciplines is discussed and analysed in this article: (1) bioinformatics, (2) therapeutic computing, (3) imaging computing, and (4) general health computing. High-performance research in bioinformatics, in particular, encourage the examination of new genome-wide infection-affiliation studies because the benefit of the healthcare field by clinical informatics is the immense amount of patient data collected for the purpose of making informed choices. In order to share clinical picture details and workflows, imaging computing is now more readily integrated with cloud services, and general health computing utilizes massive data procedures, such as Ebola, to predict and monitor irresistible disease flare-ups. In this document, we address the on-going growth and progress of broad data applications in these areas of health care and outline the obstacles, gaps and opportunities for the creation and progression of large health care data applications.

Shahzad Ahmad et al (2016),Headway in sequencing innovation has brought about the era of a broad measure of bioinformatics data that can be broken down in a limited timeframe. Custom techniques cannot be tailored to the speed and the scale of the data age. New platforms, apparatuses and procedures should be examined for the analysis of results, which might face the challenge of life. Big data devices and approaches resolve problems of size, scalability and efficiency. In this article, we present the capacity of the machine learning procedures performed by Hadoop and Spark to analyse bioinformatics results. First, we summarise the current work around there and then discuss the potential analysis of the bearings and the openings.

Simone Leo et al (2010), Bioinformatics systems actually include both the planning of giant data calculations and the weighty estimation. Satisfying these requirements calls for a clear solution to upgrading parallel computation. MapReduce is a general purpose parallelization paradigm that appears to be specifically suitable for this undertaking and for which open-source use (Hadoop) is available. Here we report on its application to three main algorithms: BLAST, GSEA, and GRAMMAR. The first is represented by a relatively low-weight estimation on broad data sets, whereas the second involves a hefty planning on typically limited data sets. The 
third may be assumed to contain a mixture of these two computational flavours. Our findings are empowering and demonstrate that the architecture will provide a broad variety of uses for bioinformatics while retaining strong computational abilities, scalability and ease of service.

Kathleenet al (2013), On bulk data measurements, there is often a need to run machine learning duties. In fields such as design recognition, data analysis, bioinformatics and suggestion systems, program activities. Here we measure the output under two different cloud runtimes, Hadoop and Granules, of 4 clustering algorithms and 2 arrangement algorithms supported by Mahout. To ensure a reasonable review, our metrics use a common Mahout back-end code. The comparisons between these uses come from the manner in which runtimes of Hadoop and Granule (1) handle and cope with the life cycle of individual calculations and (2) how they coordinate data trading throughout the incremental clustering processes between the various stages of the computational pipeline. In a distributed setting, we are combining an investigation of our results with all of these algorithms, almost like a conversation on steps of failure recovery.

Wei Dai (2014), Late years saw the advancement of distributed computation and the Big Data era, causing difficulties for traditional tree-choice algorithms. To begin with, when the scale of the dataset turns out to be very high, the way to create a tree of choice can be very cumbersome. Second, on the ground that data will no longer fit into memory, certain measurements have to be transferred to outside storage and therefore raise the expense of I/O. To this end, we propose to conduct a common option tree calculation, C4.5, using the MapReduce programming model. In specific, we turn the normal equation into a progression of the Chart and Minimize methodology. Plus, we are preparing certain data systems to reduce connectivity costs. We're also steering large analyses to a massive dataset. The findings reveal that our estimate demonstrates both time efficiency and scalability.

Shanahan et al (2014),We're addressing the applicability of bioinformatics to the Microsoft cloud computing framework, Azure. We're focused on the resource's accessibility rather than its effectiveness. An example of how a large number of microarray expression data contained in the public Array Express database can be evaluated with R on Azure. We include a walk through to clearly illustrate how Azure can be used in Appendix S1 to perform these analyses, and provide a contrast with local computing. We note that the use of the Azure Platform as a Service (PaaS) offering will present a steep learning curve for developers of bioinformatics who will usually have a history of Linux and scripting. On the other hand, the inclusion of an external library collection makes it easier to run applications in parallel (scalable) mode and to handle such a development run directly with only a few hundred lines of code, much of which can be implemented from the blueprint. This environment is recommended to be better adapted to the use of safe bioinformatics tools by consumers who are not interested in its growth.

M. A. Sarwar et al (2016), Genome sequences have been upset by cutting-edge technologies that can easily produce large volumes of data at modest ease. Now that data production has been limited, there is an enormous test to break down the data flood and interpret the biological significance. Bioinformatics researchers have reached people's high standards and various software resources and databases have been delivered and are continuing to grow with this fast-moving sector. Here, we set out a portion of instruments and databases that are widely used to analyse cutting-edge sequence data with commentary on their usefulness.

Satvik Vats et al (2020), Custom data examination apparatus is equipped to handle the deviated form of data, i.e., ordered, semi-organized and unstructured. The organised conduct of the data supplied from different suppliers includes the establishment of suitable instruments. For these devices, restricting the use of energy to manage an enormous amount of data is a measure which affects the performance of the device's implementation time. We proposed a time streamlining model along these lines in the current paper that would share standard HDFS (Hadoop Distributed File System) between three Name-hub (Master Node), three Data-hub, and one Client-hub. Within the Demilitarized Zone (DMZ), these hubs work to ensure uniformity. In a free platform, machines taking roles are studied to recognize this model. Mahout is implemented in the main hub (Name-hub 1) via expert vaults for all machine learning libraries. 


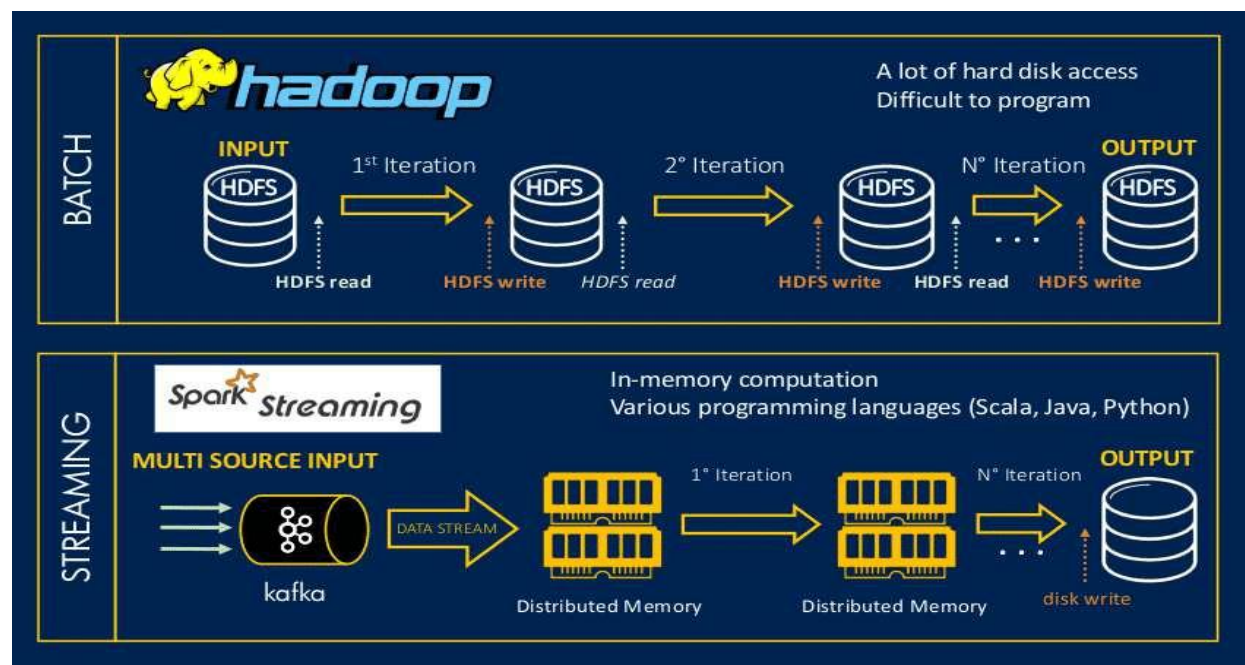

Fig 4 example hadoop system.

In this architecture streaming and batch files are observed for HDFS file reading and righting process. The memory location adjusted in HDFS system can handle the distributed memory through iterations. These iterations starting from 1, 2, N, disk writing and reading functionalities are implemented java, python and HTML languages. It is an hadoop architecture and handle a lot of file storage problems using conventional mechanism in fig 4

A sparkling worker goes into the next hub (Name-hub 2), R, linked to Hadoop. In the third hub (Name-hub 3) that is used to break down logs, Splunk is organised. In order to decide the period of reaction, time of execution and throughput, examinations are carried out between the proposed model and the inheritance model. K-means clustering, Navies Bayes, and suggested algorithms are run on three different data sets, i.e. film rating, newsgroup, and spam SMS data set, and independently interact with arranged, semi-organized, and unstructured data.The option of devices characterises the data sovereignty, e.g. the Newsgroup data collection to operate on Mahout, as most data cannot be viable. It is evident from the results of the data that the success of the proposed model gives rise to speculation that our model is struggling to impede the assets of the heritage model. Moreover, the suggested model will cope with some form of estimation of the diverse data arrangements that occur within the local organisations.

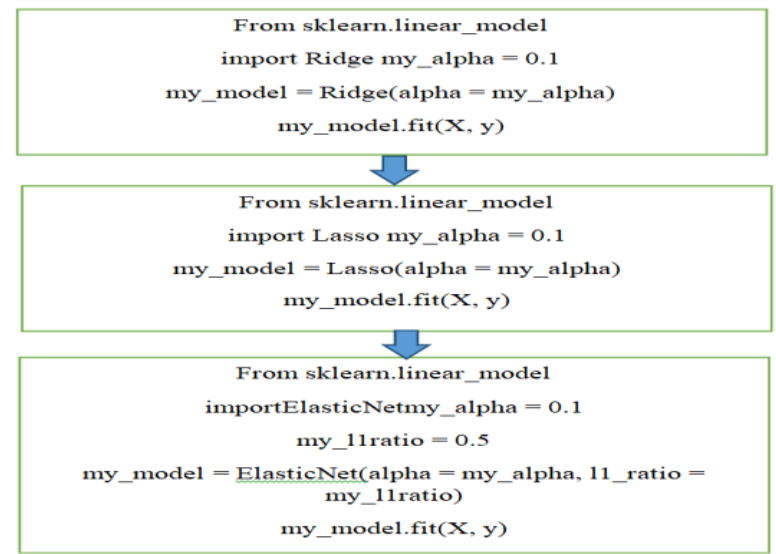

Figure 5: HDFS-ENR

Fig 5 clearly explains about elastic net regression model using lasso and ridge regression, these are solves many files storage problems with effective manner. But, have many limitations. The alpha module can solves the file shrinking problem with zero coefficient analysis. Coming to linear and non-linear models this mechanism do not reach out the current issues.

\section{Algorithm:MSR}

Input: file(any extension)

Output: reduced file, encrypted file

Map phasing:

Step: 1 portioning

Step: 2 read ()

Step: 3 tokenize ()

Step: 4 covert to MSR model

Reducing MSR: 
Step: 5 combine the files

Step: 6 count the memory

Step: 7 decisions

Step: 8 stop the process

Ready Queue of Small Files in Order of

\begin{tabular}{|l|l|l|l|l|l|l|}
\hline & FN & & $\cdot$ & $\cdot$ & & F4 \\
\hline
\end{tabular}

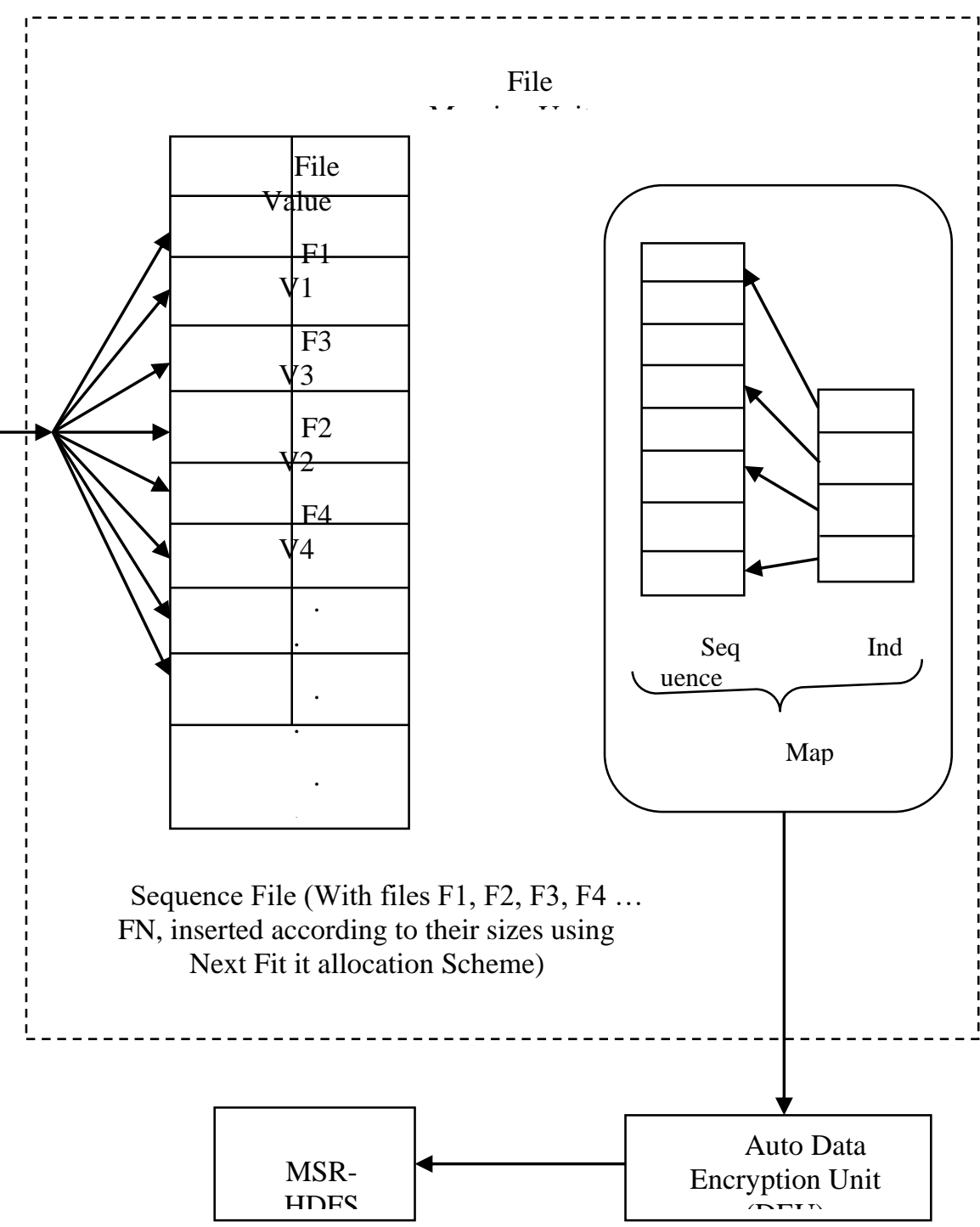

Fig5 Architecture of automatic File

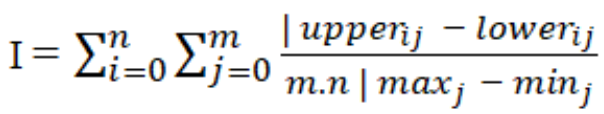

The above equation 1 calculating the upper bond and lower bond attributes using minimum and maximum file sizes. Ifi $>100 \mathrm{~kb}$ then automatically our system transfer that file into hadoop network else, store in the same server. Above figure used as a file merging technique in MSR-HDFS, in this files ant its values are clearly varied by proposed technique.

\section{Experimental setup}

Install java in $\mathrm{C}$ directory of your system and set path and java home

Put hadoop folder in $\mathrm{C}$ directory of your system and set path to C:/hadoop/bin and then set hadoop home as Variable name $=$ BIO-INFORMATICS DATA USING HADOOP_HOME

Variable value $=\mathrm{C}$ :/bio-informatics data using hadoop

Follow below instructions to run bio-informatics data using hadoop server

Go cd/bio-informatics data using hadoop/bin and execute command 
hdfs namenode -format

Now set location in command prompt

C:/bio-informatics data using hadoop/sbin and run command start-dfs

Above command will start hadoop server

Then open browser and enter urlhttp://127.0.0.1:50070/dfshealth.jsp to get below hadoop home page

\section{NameNode 'localhost:9000' (active)}

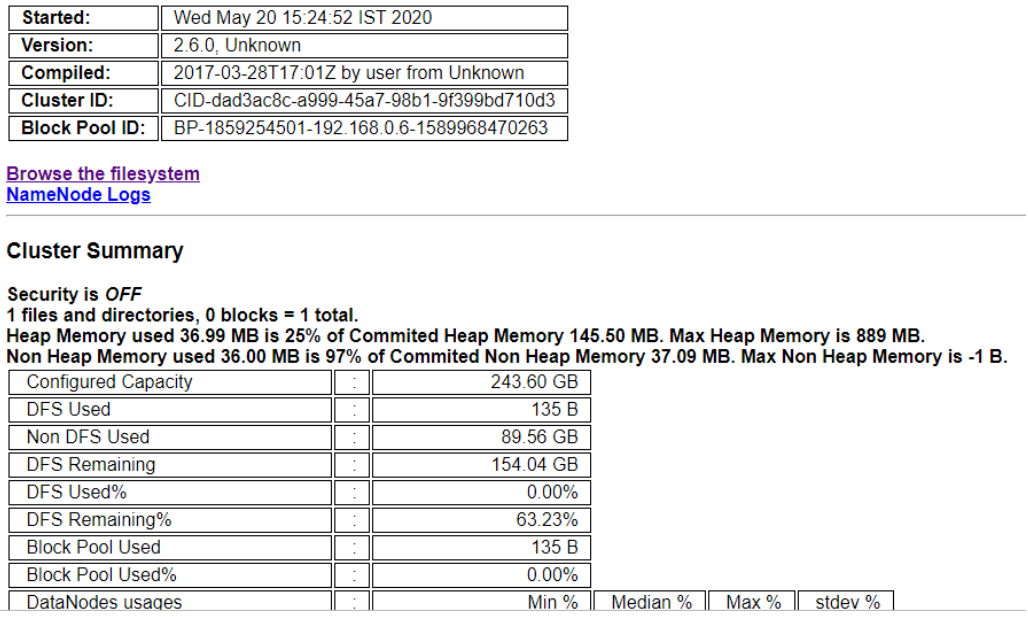

Figure: 6. Namenode allocation

Now create Python folder in your system C directory and then put 'DynamicMerging' folder in C:/Python folder. Above fig. 6 shows the Namenode point related to local host system.

Open console and start DJANGO server using below command

$\mathrm{C}$ :/Python/DynamicMerging and run command as 'python manage.py runserver'

Now open browser and enter URL 'http://localhost:8000/index.html to get below page

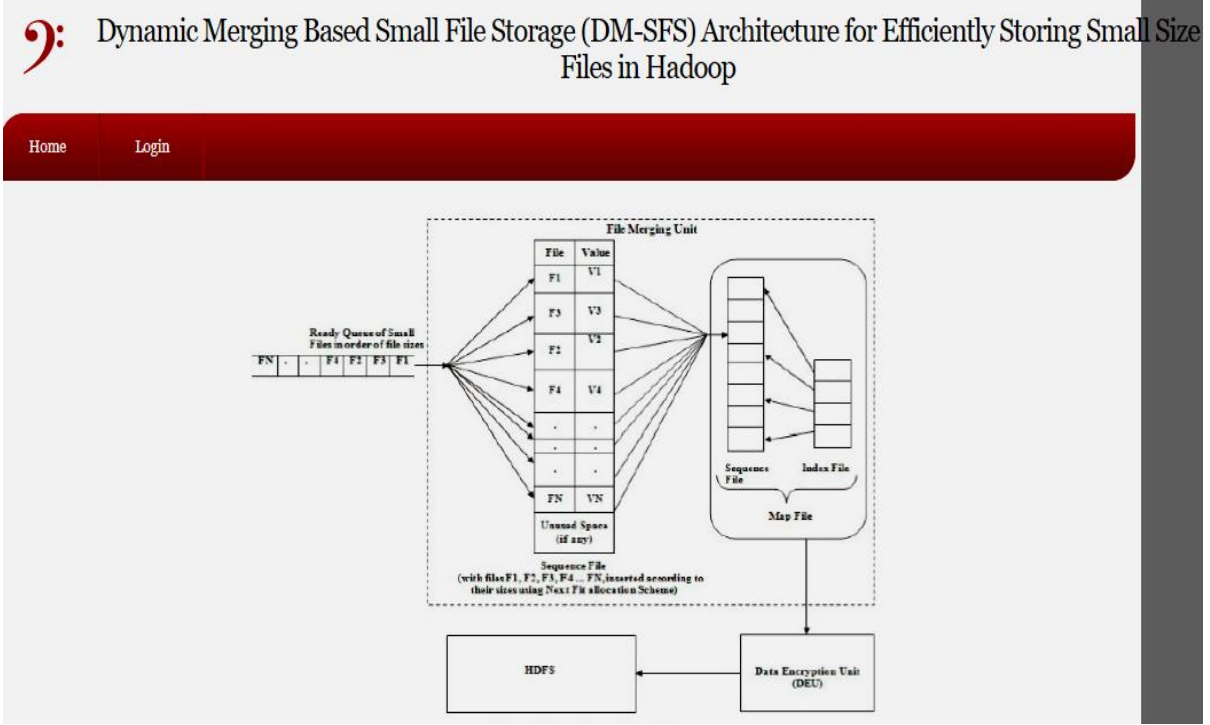

Figure: 7.HDFS-MSR-ESR

The fig.7 explains about, automatic file managing unit for MSR-ENR in HDFS system. It can handle the issues effectively with elastic net regression model. It is a machine learning engine, there are entire analysing is handle with cluster memory. In above screen click on Login link to get below screen, using credentials users are entire into environment ofbio-informatics data using Hadoop. 


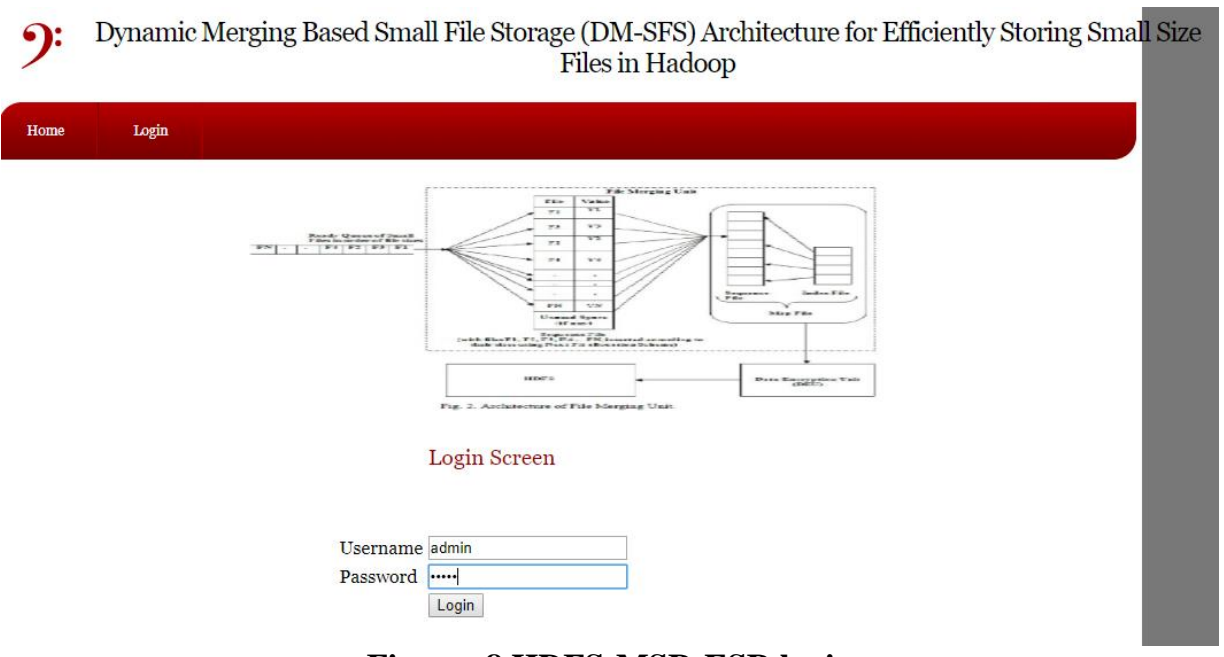

Figure: 8.HDFS-MSR-ESR login

Above figure. 8 describe about, Hadoop entry place in this username and password is necessary. ** User id: Admin, ** password: Admin, utilizing this we can sort out the files clearly. In above screen enter username as 'admin' and password as 'admin' to get below screen ): Dynamic Merging Based Small File Storage (DM-SFS) Architecture for Efficiently Storing Small Size Files in Hadoop

\section{Upload Fle To Hadoop Download Fle From Hadoop $\quad$ Logout}

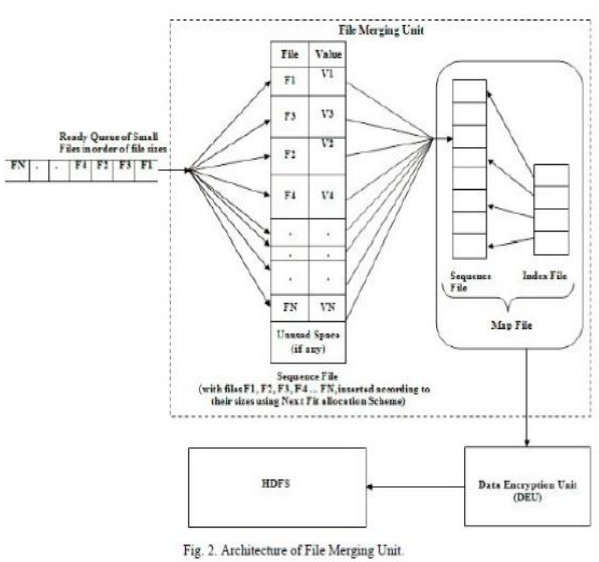

Figure: 9. File upload

In above screen click on 'Upload File to bio-informatics data using Hadoop' link to upload file to bioinformatics data using hadoop, fig.9 explains about upload and verify options of ENR technology. After uploading when the file size more than $100 \mathrm{~kb}$, then automatically transfer this into HDFS location. The encoding process is performed on this same file for less storage activation. 


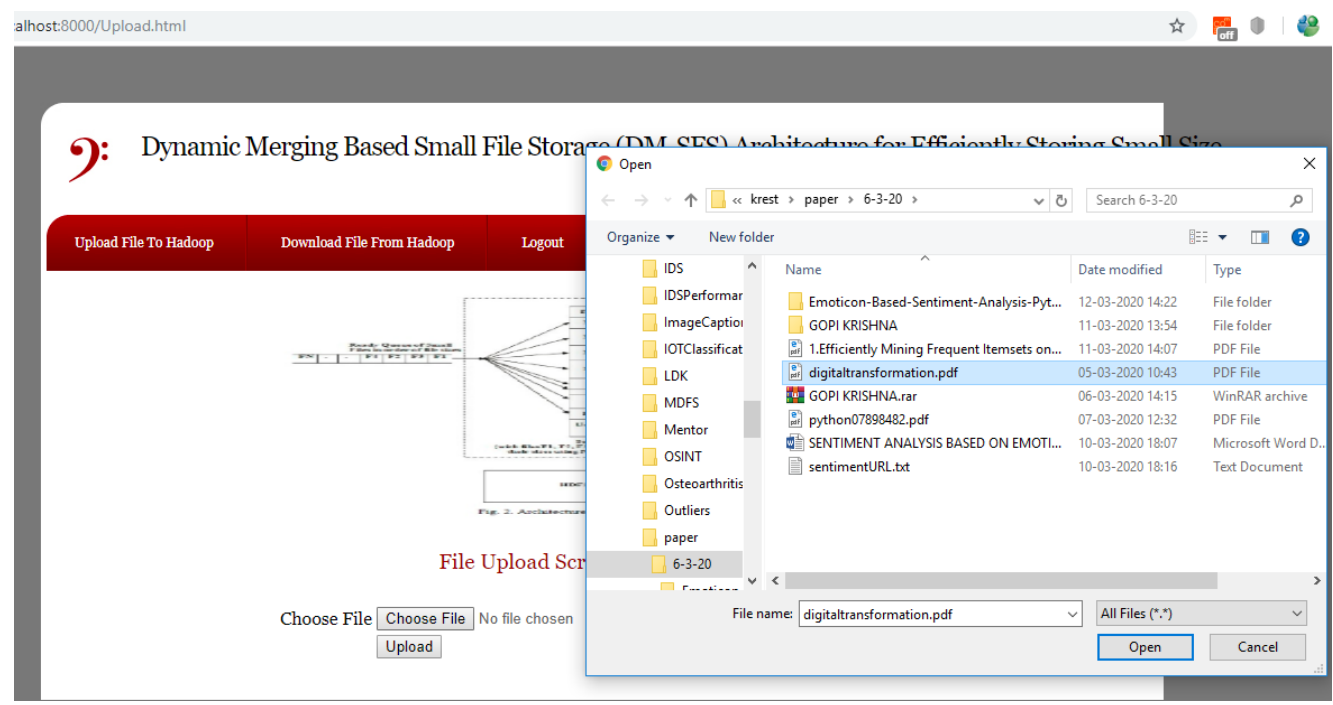

Figure: 10 Uploading folder

In above screen we uploading one pdf and now click on 'Open' button and then click on 'Upload' button to store file at bio-informatics data using hadoop, which is shown in fig. 10

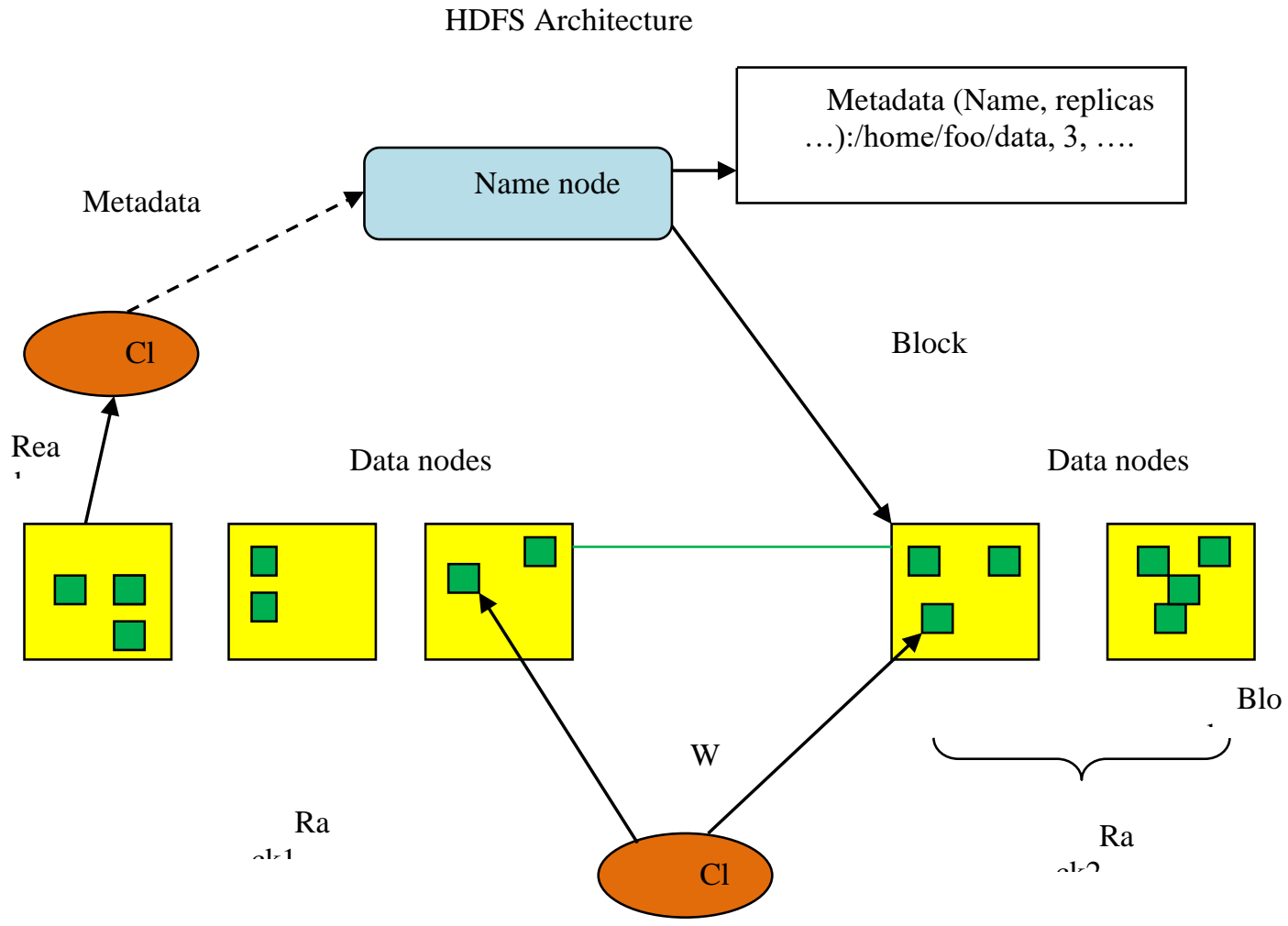

Figure: 11 data node and name nodes

Above fig.11 explains about exact process of name nodes and data nodes, it is identified that block operations placed separately. The data node and name nodes are collected for HDFS function verification. 


\section{Results and discussion}

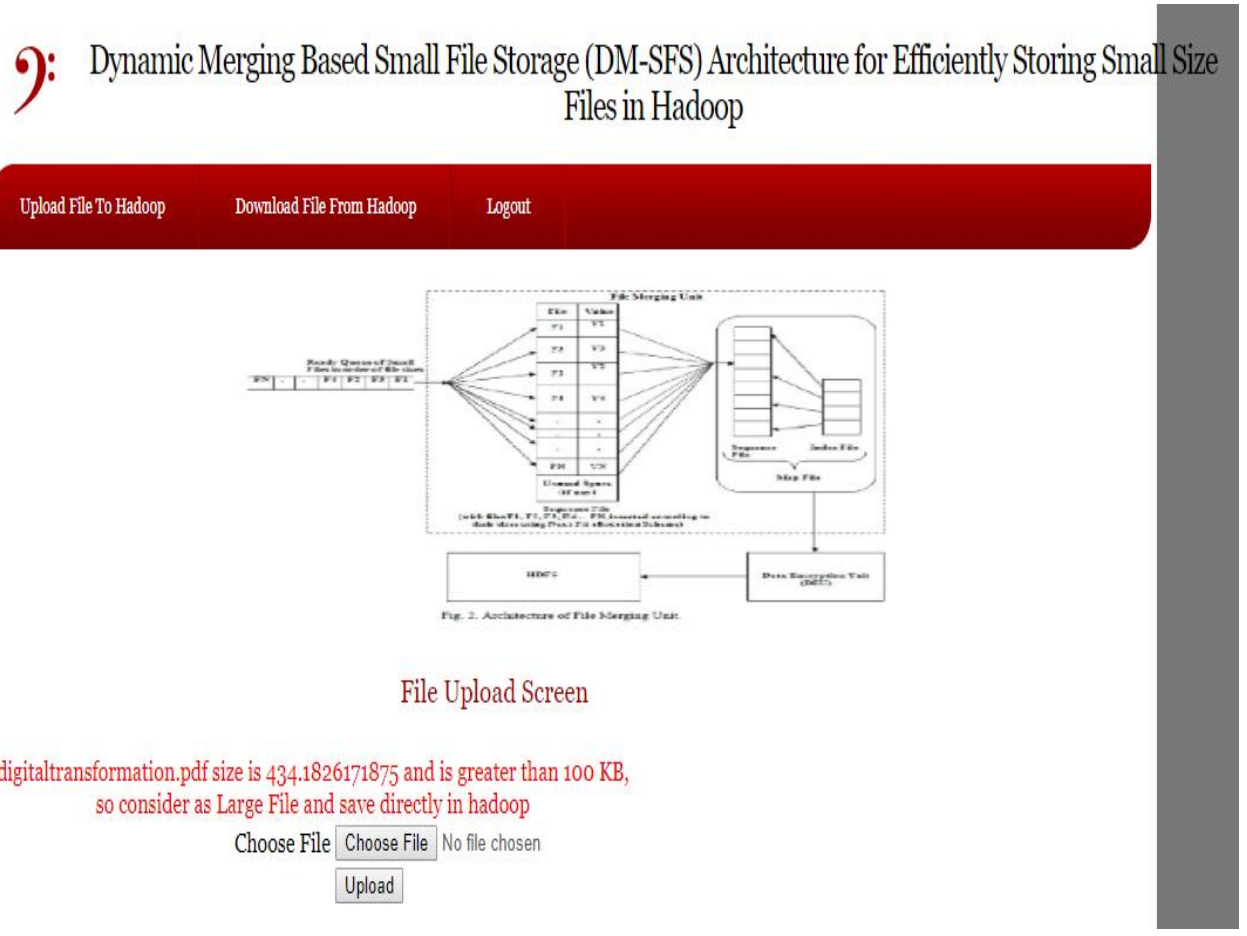

Figure: 12. Results of file

In above screen pdf file size is $434 \mathrm{~KB}$ which is greater than $100 \mathrm{~KB}$ so it will consider as large file and store directly atbio-informatics data using hadoop and before storage file will be encrypted using two fish algorithm. Now can see file in hadoop server by using this URL 'http://127.0.0.1:50070/dfshealth.jsp' shown in fig.12

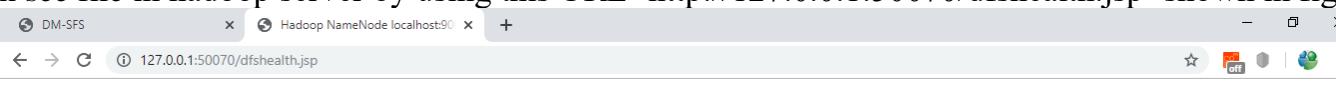

\section{NameNode 'localhost:9000' (active)}

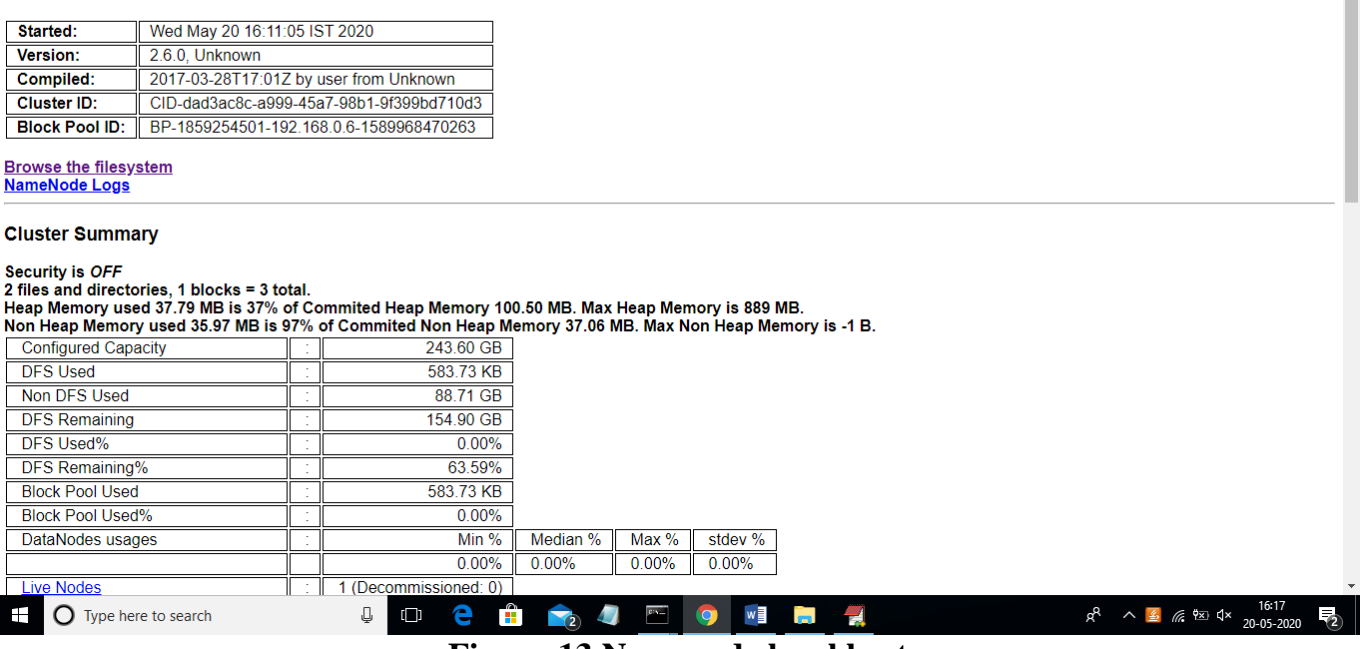

Figure:13 Namenode local host

In above screen click on 'Browse the Filesystem' link to view files in below screen, show in fig. 13, these are explaining the date, time, cluster id and block pool id. This type of data can handle the system make efficient for future file handling mechanism. 


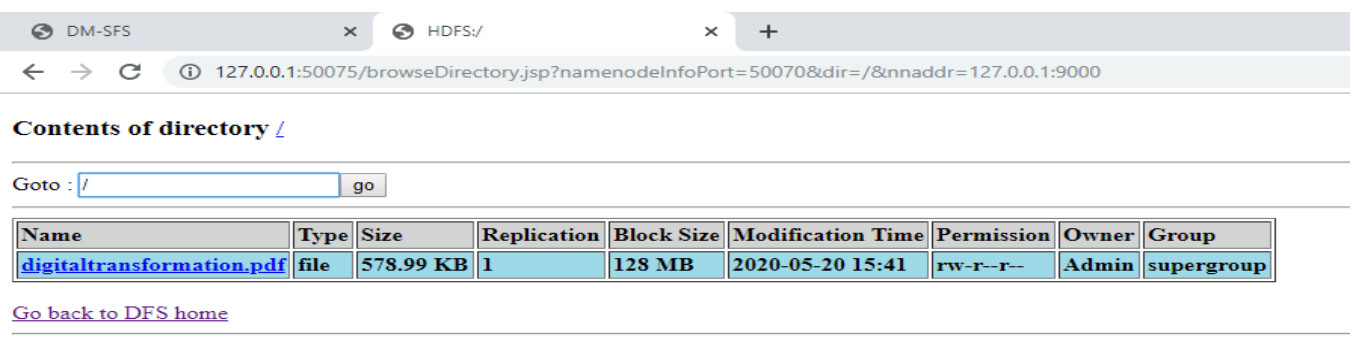

\section{Local logs}

Log directory

Hadoop, 2020.

Figure: 14. Uploaded files

In above screen we can see file name which we upload from application is store at hadoop server and now click on that file name to see it content as encrypted shown in fig14

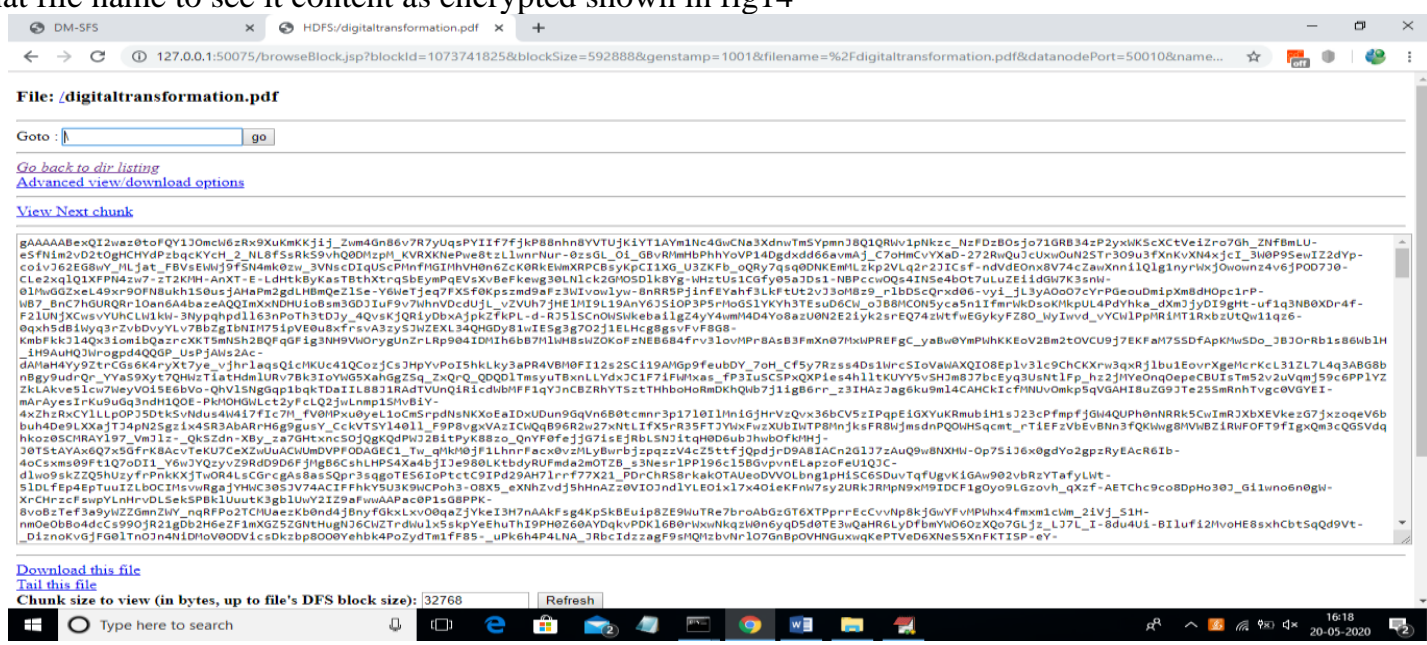

Figure: 15. Pdf file verification

In above screen we can see pdf file is encrypted and now go back to application and store small file and then we can see inbio-informatics data using hadoop those small files will merge in single sequence file show in fig 15

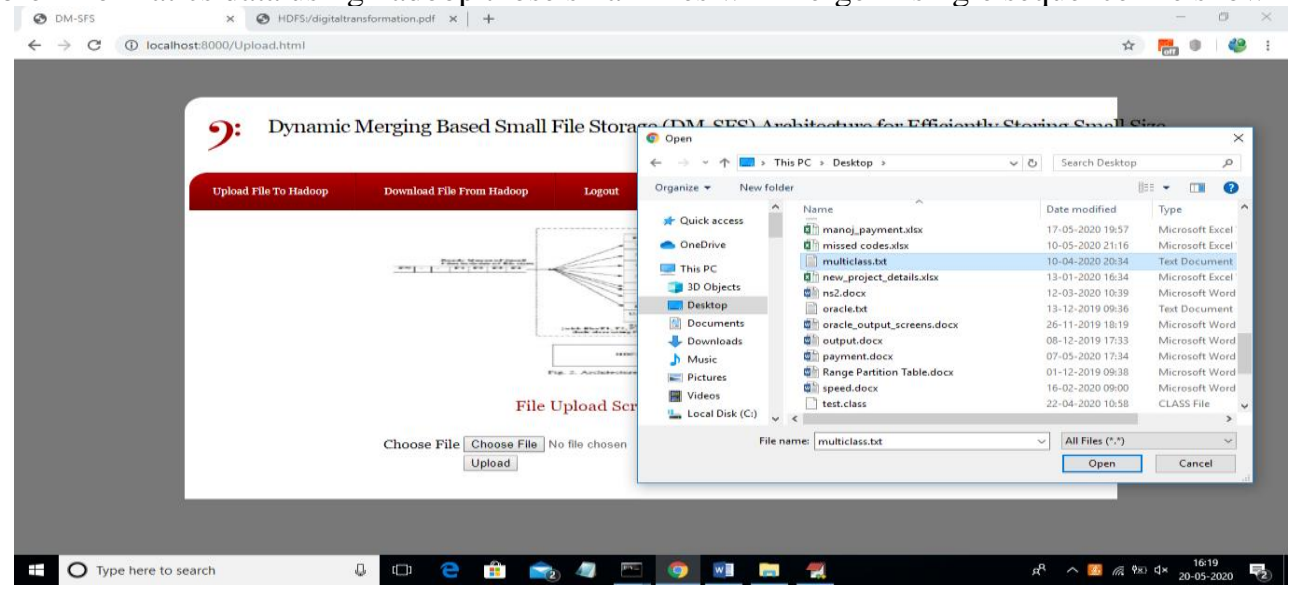

Figure: 16: small file uploading

In above screen we are selecting one small txt file, it has size if below $100 \mathrm{~kb}$ means hadoop directly allotted this in this general workspace which is shown in fig16 


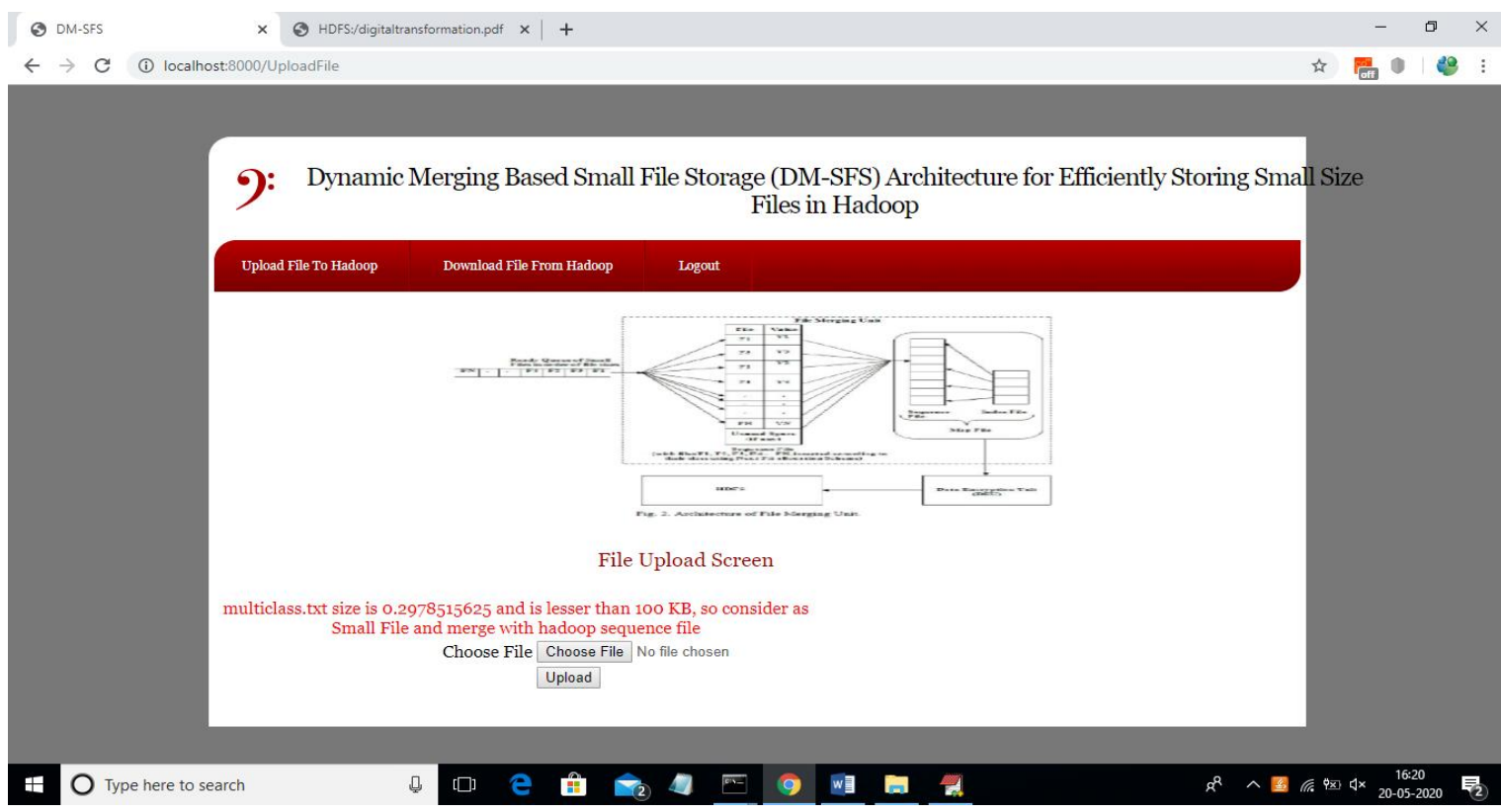

Figure: 17.Results of small size file

In above screen multiclass.txt file is a small file and it will merge in sequence file and now again we will upload one more small file, for further action recognition in fig 17

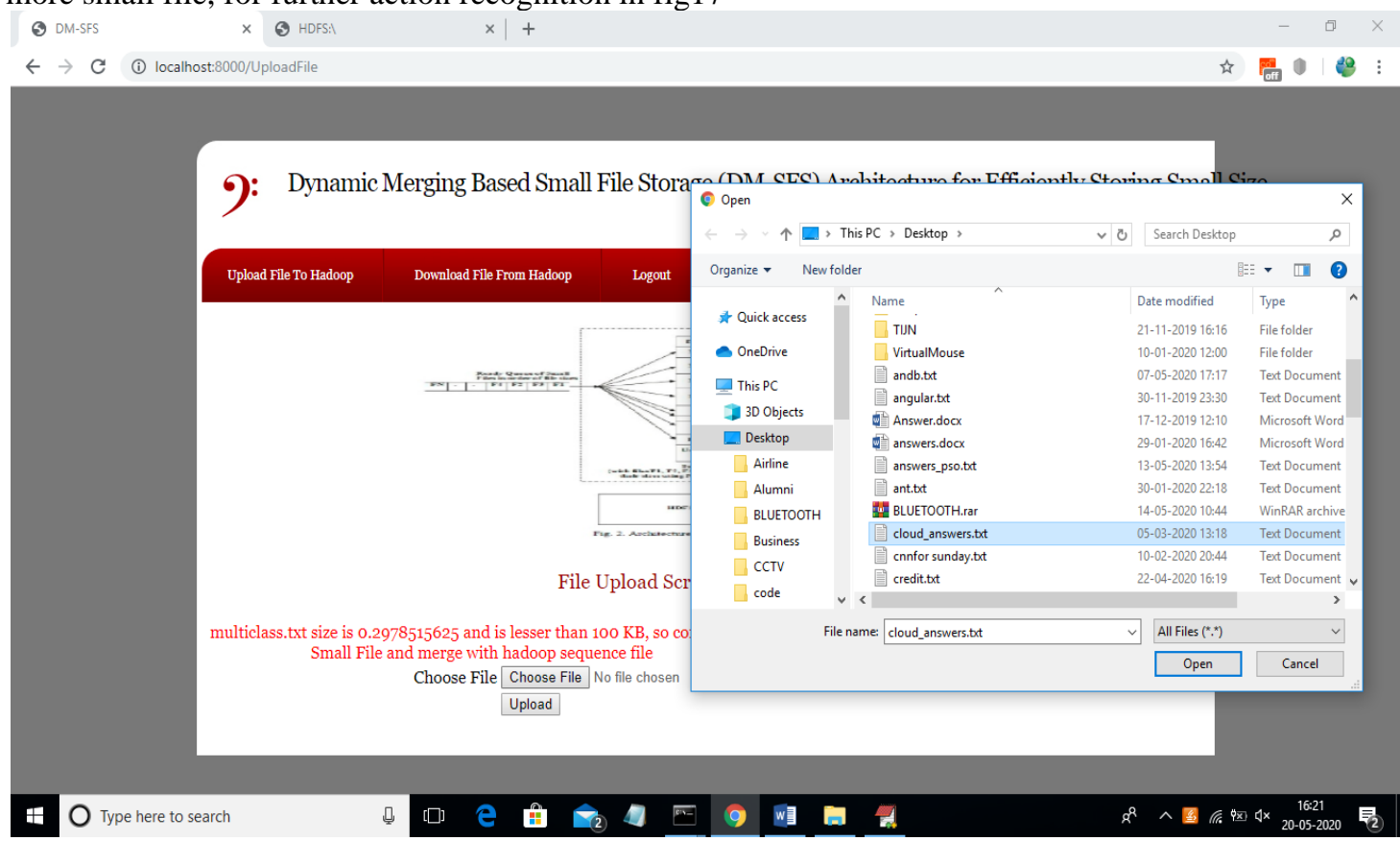

Figure: 18Results of small size file

In above screen we are uploading one more txt file and below is the storage result, it is identified that upload file is small in size in fig 18 


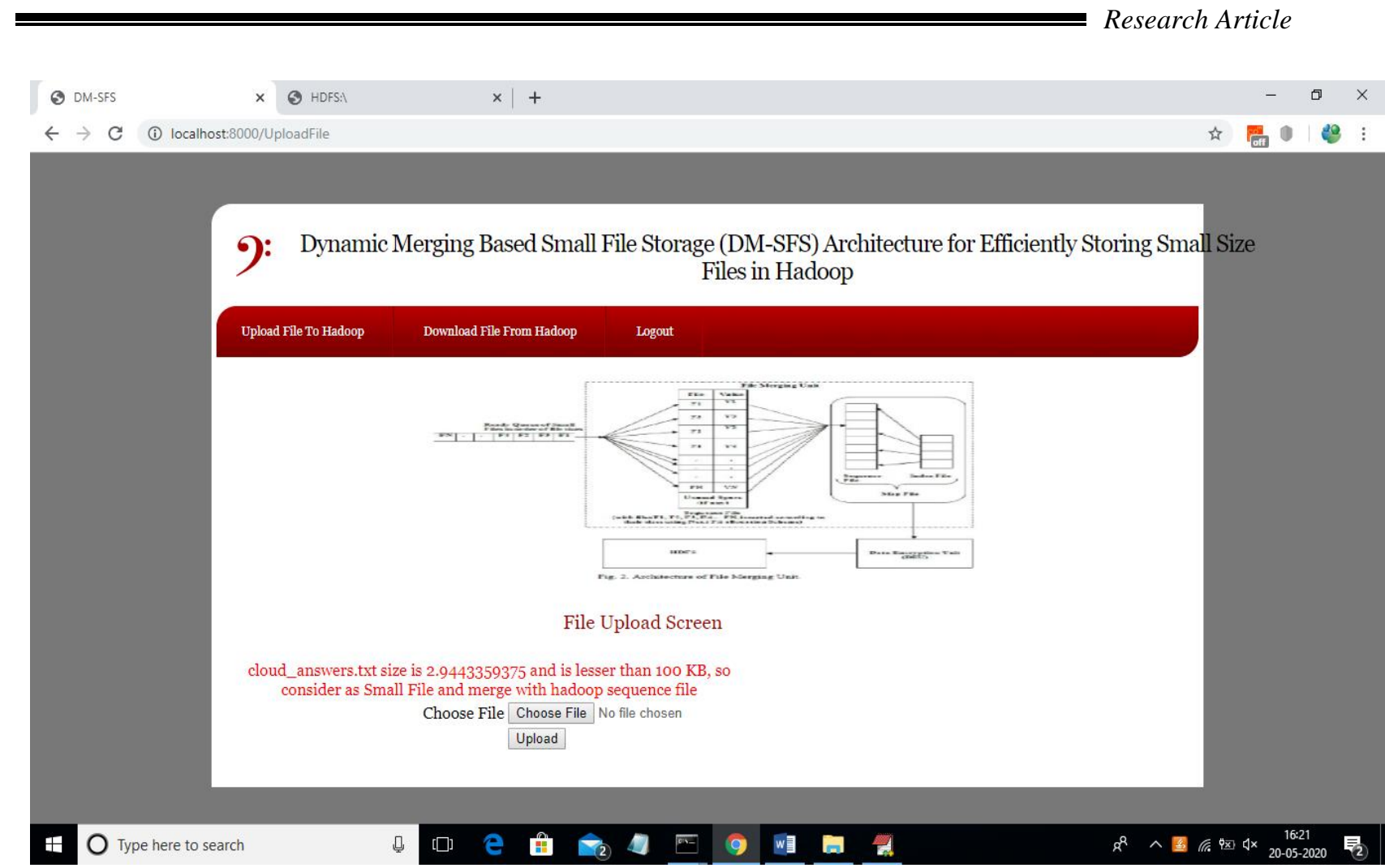

\section{Figure: 19. Small file message}

In above screen clouod_answers.txt is also small file and will be merge in sequence file and now we can see atbio-informatics data using hadoop server all store files shown in fig. 19
(5) DM-SFS
(5) HDFS:
$\leftarrow \rightarrow$ C (i) 127.0.0.1:50075/browseDirectory.jsp?namenodelnfoPort $=50070 \&$ dir $=/$ \&nnaddr $=127.0 .0 .1: 9000$

\section{Contents of directory $\angle$}

\begin{tabular}{|c|c|c|c|c|c|c|c|c|}
\hline \multicolumn{2}{|l|}{ Goto : 1} & \multicolumn{7}{|c|}{ go } \\
\hline Name & Type & Size & \begin{tabular}{|l|} 
Replication \\
\end{tabular} & Block Size & Modification Time & \begin{tabular}{|l|} 
Permission \\
\end{tabular} & Owner & Group \\
\hline digitaltransformation.pdf & file & $578.99 \mathrm{~KB}$ & 1 & $128 \mathrm{MB}$ & $2020-05-2015: 41$ & rww-r--r-- & Admin & supergroup \\
\hline sequence.pkl & file & $4.57 \mathrm{~KB}$ & 1 & $128 \mathrm{MB}$ & 2020-05-20 16:21 & rww-r--r-- & Admin & supergroup \\
\hline
\end{tabular}

Go back to DFS home

\section{Local logs}

Log directory

Hadoop, 2020

\section{Figure: 20.content directory}

In above screen we can see one pdf file and one sequence file and actually we upload one pdf file and two small txt file but small txt file merge inside sequence.pkl file so we got only 1 file for two small files. Now go back to application and download those files by clicking on 'Download File frombio-informatics data using Hadoop' link shown in fig. 20 


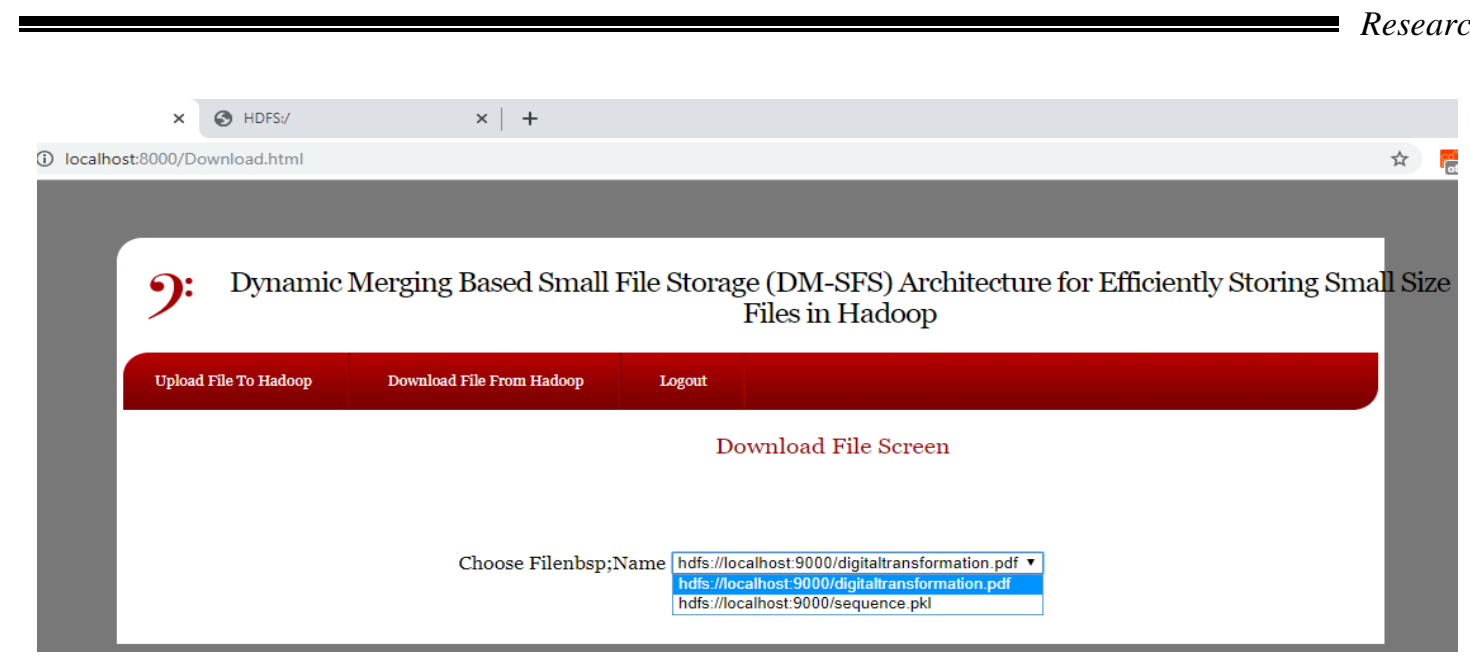

Figure: 21Download screen

In above screen all uploaded files we can see in drop down box and then we can select any file and that file will download inside 'C:/bio-informatics data using HadoopDownload' folder. Show in fig.21

$\times$ 6 HDFS: $\quad \times 1+$

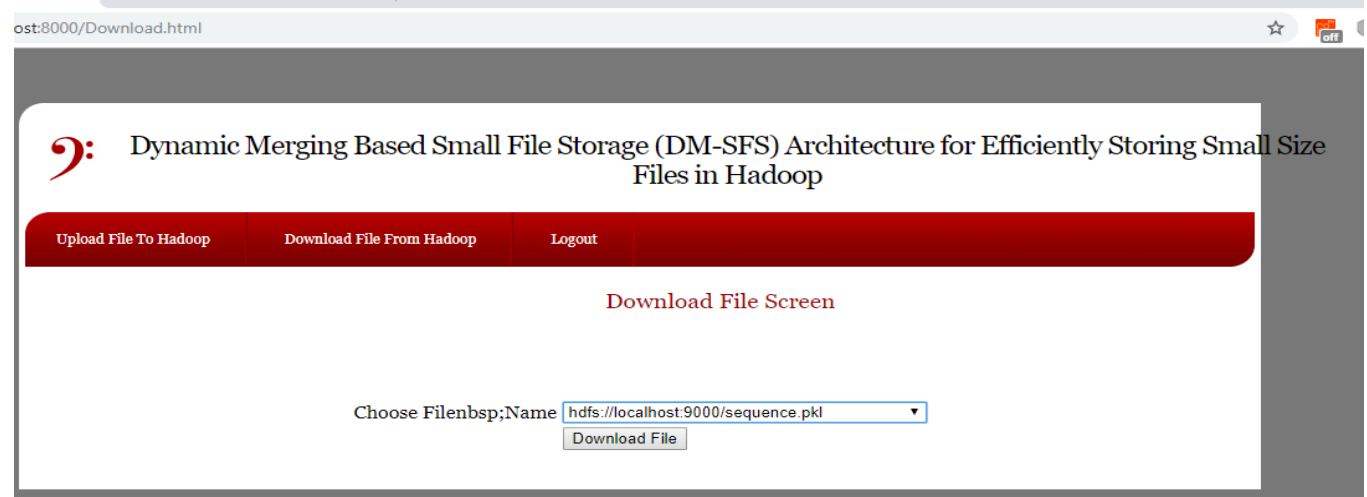

\section{Figure: 22. Downloaded file}

In above screen we are selecting sequence.pkl file which contains merge of two file and those two files will be downloaded in C:/bio-informatics data using HadoopDownload folder shown in fig 22
$\times$ (5) HDFS:
$x \mid+$

(i) localhost:8000/DownloadFile

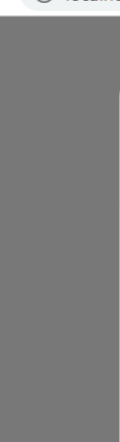

Dynamic Merging Based Small File Storage (DM-SFS) Architecture for Efficiently Storing Small Size Files in Hadoop

\section{\begin{tabular}{ll|l}
\hline Upload File To Hadoop $\quad$ Download Flie From Hadoop $\quad$ Logou \\
\hline
\end{tabular}}

Download File Screen

File downloaded in C:/HadoopDownload folder

Figure: 23. Location of folder

In above screen we can see file downloaded inside C:/bio-informatics data using HadoopDownload' folder and now we check that folder shown in fig 23 


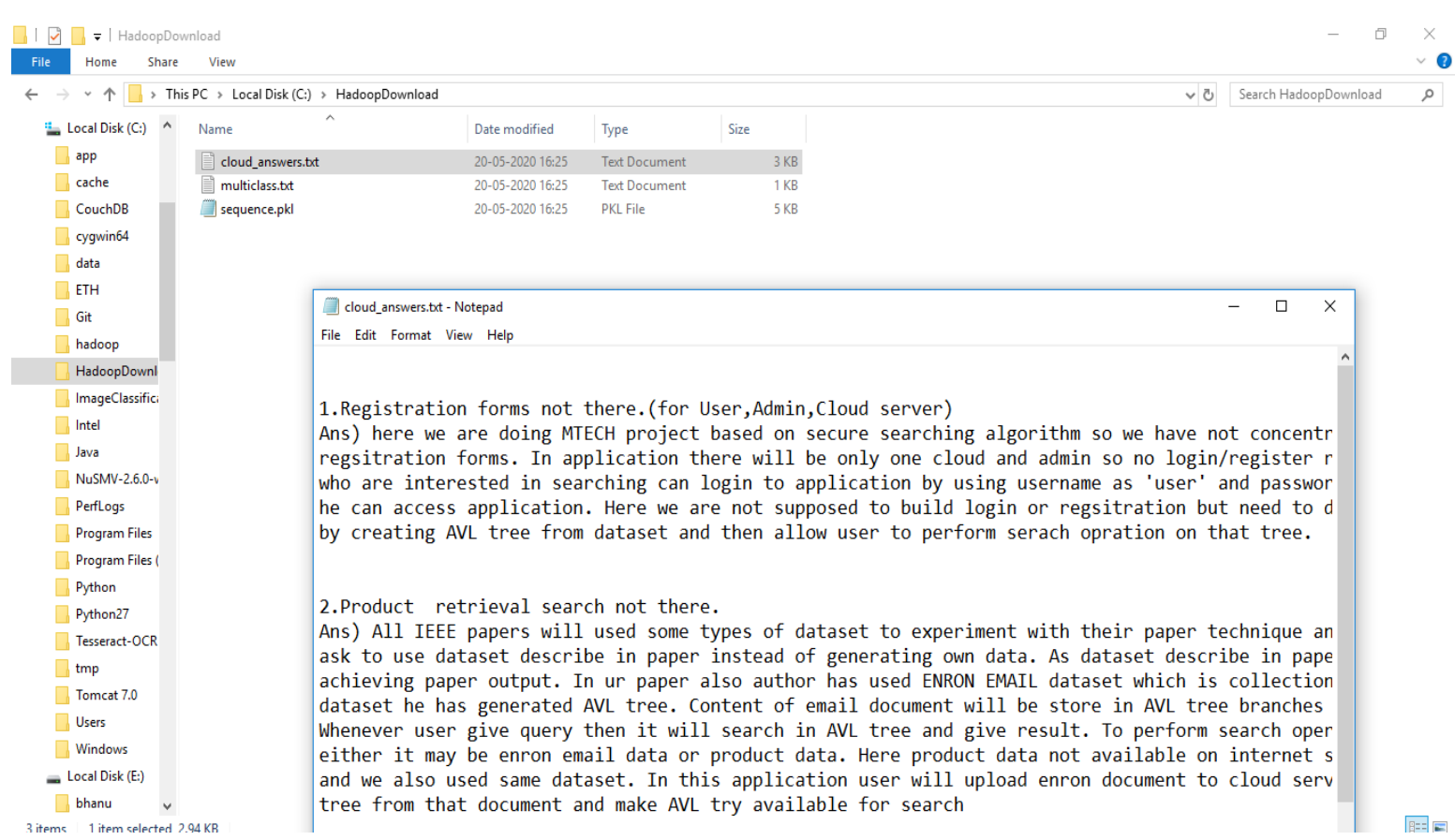

Figure: 24Merge File

In above screen we can see complete sequence.pkl and all its merge files downloaded and decrypted successfully. Same sequence file u can see in hadoop server in encrypted format in below screen shown in fig 24

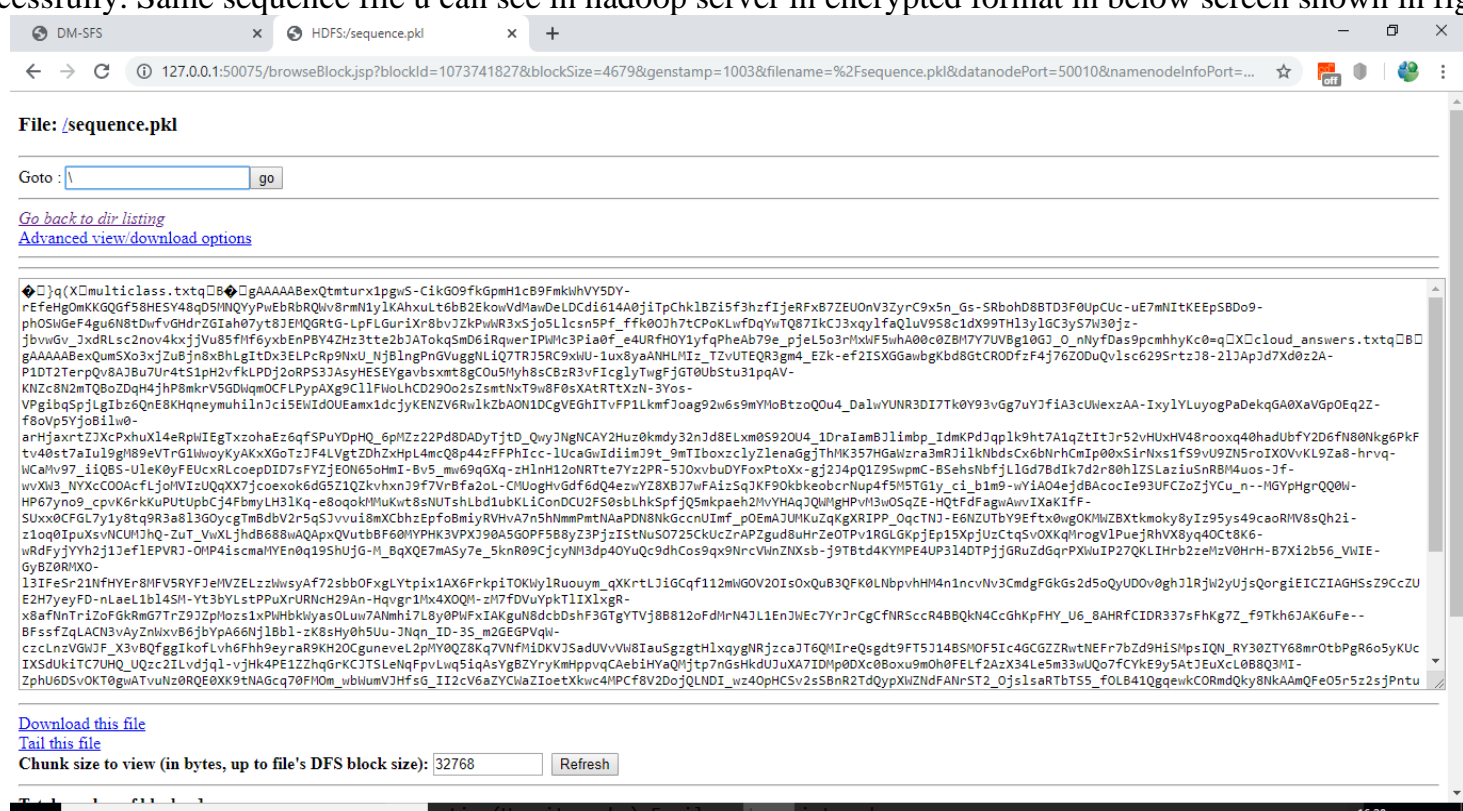

Type here to search

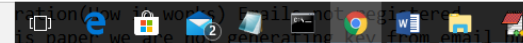

Figure: 25encrypted format

In above screen at hadoop server content of sequence.pkl is in encrypted format, it is clearly shown in above fig. 22 explains that encryption format of file shown in fig 25

Table: 1 comparison of results

\begin{tabular}{|l|l|l|l|l|l|}
\hline methods & Accuracy & Sensitivity & Specificity & Recall & Through put \\
\hline Misclassification[15] & 74.23 & 57.67 & 85.39 & 81.42 & 74.9 \\
\hline HDFS[16] & 74.83 & 60.07 & 84.77 & 72.58 & 79.1 \\
\hline H+S [17] & 74.75 & 61.00 & 84.02 & 83.45 & 81.45 \\
\hline H+S+C [17] & 77.06 & 71.23 & 81.34 & 84.78 & 82.68 \\
\hline $\begin{array}{l}\text { HDFS+MSR+ENR } \\
\text { Proposed }\end{array}$ & 94.76 & 97.87 & 98.75 & 94.58 & 92.48 \\
\hline
\end{tabular}




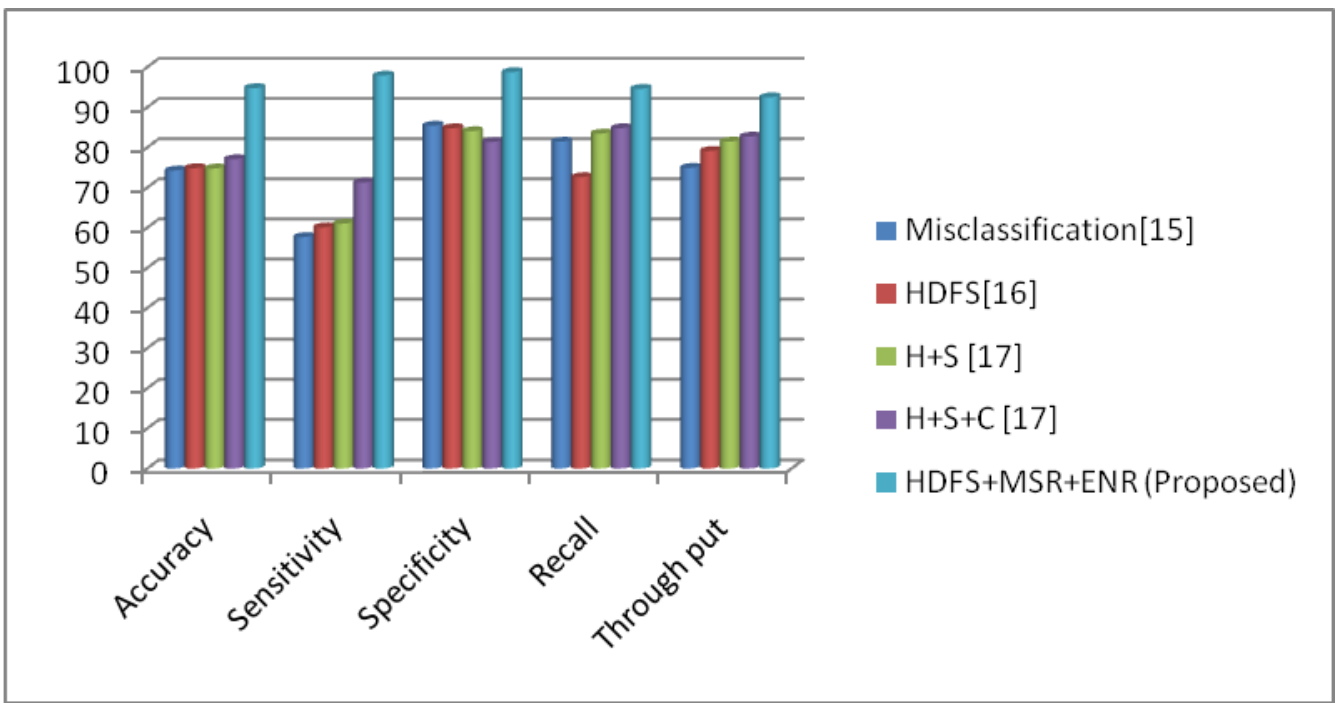

Figure: 26. Comparison of results

Figure: 26 and table .1 explains about entire performance measures related existed and proposed method MSR+ENR, in this clearly explains about proposed method achieves more improvement

Table: 2. Processing time

\section{Processing Time}

\begin{tabular}{|l|l|}
\hline Methods & Processing Time in ms \\
\hline Misclassification[15] & 7200 \\
\hline HDFS[16] & 5800 \\
\hline H+S [17] & 800 \\
\hline H+S+C [17] & 700 \\
\hline $\begin{array}{l}\text { HDFS+MSR+ENR } \\
\text { Proposed }\end{array}$ & 420 \\
\hline
\end{tabular}

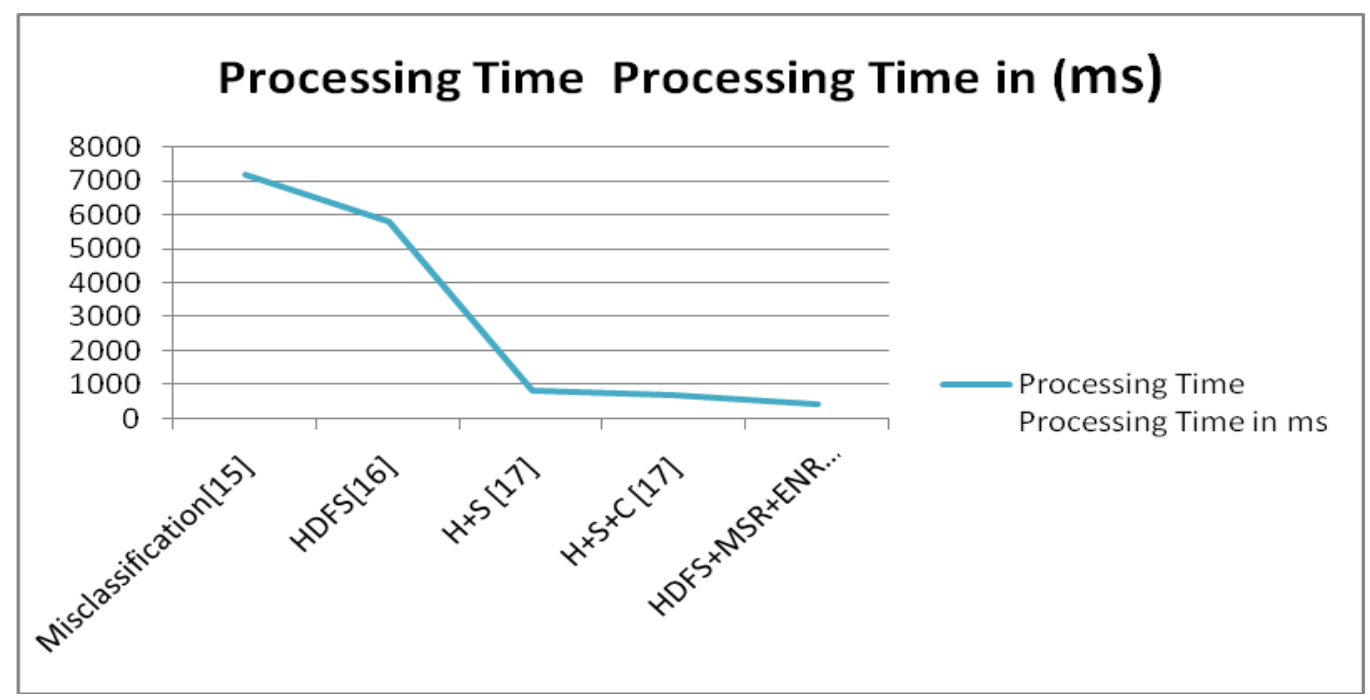

Figure: 27Processing time

Figure .27 and table :2 explains about processing time allocation to particular method, in this proposed method MSR+ENR achieves more improvement compared to existed methods.

\section{CONCLUSION}


The development of cutting edge sequencing innovation has been coordinated by a parallel development in related bioinformatics tools. These tools keep on expanding in number and complexity. It is significant that clients know about the contrasts between clearly comparable tools in the event that they are to settle on educated choices and embrace the most proper examination. As can be seen by the vast number of projects previously depicted, Hadoop and its associated open source projects provide a distinct and developing bioinformatics network of both clients and designers. Dean and Ghemawat pursue a finishing stage, excluded from the beginning work for the Hadoop/HBase-based PNNL project. That is, the scalability allowed by Hadoop and HBase is not exclusively essential for much bioinformatics work, but the simplicity of incorporating and analyzing numerous broad, dissimilar data sources into one data storage room under Hadoop, usually hardly any HBase tables, is also important.

\section{REFERENCES}

1. J. Luo, M. Wu, D. Gopukumar and Y. Zhao, "Big Data Application in Biomedical Research and Health Care: A Literature Review,” Biomedical Informatics Insights, vol. 8, pp. 1-10, 2016.

2. R. C. Taylor, "An overview of the Hadoop/MapReduce/HBase framework and its current applications in bioinformatics," in Bioinformatics Open Source Conference (BOSC), Boston, MA, USA, 2010.

3. M. U. Ali, S. Ahmad and J. Ferzund, "Harnessing the Potential of Machine Learning for Bioinformatics using Big Data Tools,” International Journal of Computer Science and Information Security (IJCSIS), 2016.

4. N. Abdulla , S. G. Nair, Z. A. M. Gazzali and A. Khade, "Measure Customer Behaviour using C4.5 Decision Tree Map Reduce Implementation in Big Data Analytics and Data Visualization," International Journal for Innovative Research in Science \& TechnologyIJIRST, vol. 1, no. 10, pp. 228-235, March 2015.

5. R. M. Esteves, C. Rong and R. Pais, "K-means clustering in the cloud - a Mahout test," in Advanced Information Networking and Applications, WAINA, 2011.

6. A. Stupar, S. Michel and R. Schenkel, "Rank Reduce - Processing KNearest Neighbor Queries on Top of MapReduce," in 8th Workshop on Large-Scale Distributed Systems for Information Retrieval, Geneva, Switzerland, 2010.

7. H. S. Yugang Dai, "The naive Bayes text classification algorithm based on rough set in the cloud platform," Journal of Chemical and Pharmaceutical Research, vol. 6, no. 7, pp. 1636-1643, 2014.

8. X.-W. C. KUNLEI ZHANG, "Large-Scale Deep Belief Nets With MapReduce," IEEE Access, 2014.

9. S. P. Kathleen Ericson, "On the performance of high dimensional data clustering and classification algorithms," Future Generation Computer Systems, 2013.

10. M. A. Sarwar, A. Rehman and J. Ferzund, "Database Search, Alignment Viewer and Genomics Analysis Tools: Big Data for Bioinformatics," International Journal of Computer Science and Information Security (IJCSIS), vol. 14, no. 12, 2016. 